\title{
Sensitivity of human papillomavirus-positive and -negative oropharyngeal cancer cell lines to ionizing irradiation
}

\author{
STEFAN HOLZHAUSER ${ }^{1,2}$, EVELYNE PIROTTE ${ }^{1}$, JOANNE JONES $^{1}$, DAVID OWENS $^{3}$, \\ ALI AL-HUSSAINI ${ }^{3}$, PETER GILES ${ }^{4}$, MERERID EVANS $^{5}$, STEPHEN MAN $^{6}$ and NED POWELL ${ }^{1}$ \\ ${ }^{1}$ HPV Research Group, Cardiff University, School of Medicine, Cardiff CF14 4XN, UK; \\ ${ }^{2}$ Department of Oncology-Pathology, Karolinska Institute, 17177 Stockholm, Sweden; ${ }^{3}$ Ear Nose/Throat/Head and \\ Neck Surgery Department, University Hospital of Wales, Cardiff and Vale University Health Board, Cardiff CF14 4XW; \\ ${ }^{4}$ Wales Gene Park, Cardiff University, Cardiff CF14 4XN; ${ }^{5}$ Velindre Cancer Centre, Cardiff CF14 2TL; \\ ${ }^{6}$ Division of Cancer and Genetics, Cardiff University, School of Medicine, Cardiff CF14 4XN, UK
}

Received February 25, 2020; Accepted June 23, 2020

DOI: 10.3892/or.2020.7709

\begin{abstract}
Human papillomavirus-positive $\left(\mathrm{HPV}^{+}\right)$oropharyngeal squamous cell carcinoma (OPSCC) has increased in incidence and has a much better prognosis than HPV-negative (HPV) OPSCC with radiotherapy alone, but exactly why is unknown. The present study therefore aimed to further examine the sensitivity and possible changes in gene expression of several $\mathrm{HPV}^{+}$and $\mathrm{HPV}^{-}$OPSCC, including various novel cell lines, upon ionizing irradiation (IR). Previously established $\mathrm{HPV}^{+} \mathrm{UM}-\mathrm{SCC}-47$, UPCI-SCC-90, CU-OP-2, CU-OP-3 and HPV- UM-SCC-4, UM-SCC-6, UM-SCC-74a, UM-SCC-19 and newly established CU-OP-17 and CU-OP-20, characterised here, were subjected to 0-6 Gy. Surviving fractions of each cell line were tested by clonogenic assays, and irregularities in cell cycle responses were examined by flow cytometry, while changes in gene expression were followed by mRNA sequencing. $\mathrm{HPV}^{+}$OPSCC cell lines showed greater variation in sensitivity to ionizing irradiation (IR) and tended to be more sensitive than HPV- OPSCC cell lines. However, their IR sensitivity was not correlated to the proportion of cells in G2 arrest, and HPV cell lines generally showed lower increases in G2 after IR. Upon IR with 2 Gy, mRNA sequencing revealed an increase in minor HPV integration sites in $\mathrm{HPV}^{+}$cell lines, and some changes in gene expression
\end{abstract}

Correspondence to: Dr Stefan Holzhauser, Department of Oncology-Pathology, Karolinska Institutet, Akademiska stråket, 17177 Stockholm, Sweden

E-mail: stefan.holzhauser@ki.se

Dr Ned Powell, HPV Research Group, Cardiff University, School of Medicine, Heath Park, Cardiff CF14 4XN, UK

E-mail: powellng@cardiff.ac.uk

Key words: human papillomavirus, oropharyngeal cancer, ionizing irradiation, clonogenic assay, mRNA sequencing, novel oropharyngeal squamous cell carcinoma cell lines in OPSCC cell lines, but not primarily those associated with DNA repair. To conclude, $\mathrm{HPV}^{+}$OPSCC cell lines showed greater variation in their sensitivity to IR, with some that were radioresistant, but overall the $\mathrm{HPV}^{+} \mathrm{OPSCC}$ group still tended to be more sensitive to IR than the HPV- OPSCC group. In addition, $\mathrm{HPV}^{+} \mathrm{OPSCC}$ lines were more frequently in $\mathrm{G} 2$ as compared to $\mathrm{HPV}^{-}$cell lines, but the increase in $\mathrm{G} 2$ arrest upon IR in $\mathrm{HPV}^{+}$OPSCC was not correlated to sensitivity to IR. Increases in minor HPV integration sites and changes in gene expression were also demonstrated after irradiation with $2 \mathrm{~Gy}$.

\section{Introduction}

The incidence of human papillomavirus-positive $\left(\mathrm{HPV}^{+}\right)$ tonsillar and base of tongue squamous cell carcinomas (TSCC/BOTSCC), the major subsites of oropharyngeal squamous cell cancer (OPSCC), (but not other OPSCC subsites) is still increasing in most western countries (1-4). Together they account for an increasing proportion of head and neck squamous cell carcinomas (HNSCC), especially in countries where smoking, a main HNSCC risk factor is decreasing $(4,5)$.

Patients with $\mathrm{HPV}^{+}$TSCC/BOTSCC usually respond well to treatment and have better long-term survival compared to $\mathrm{HPV}^{-}$TSCC/BOTSCC and HNSCC in general, irrespective if they receive radiotherapy alone, or radio-chemotherapy (i.e. 80 vs. $40-50 \%$ 5-year overall survival) (1,5-9). However, over recent decades, treatment for $\mathrm{HNSCC}$, as well as for $\mathrm{HPV}^{+}$ TSCC/BOTSCC, has gradually been intensified with radiotherapy and induction or concomitant chemotherapy, and the numbers of side effects have increased (5). Most likely this intensified therapy could be de-escalated for most patients with $\mathrm{HPV}^{+}$TSCC/BOTSCC, but to facilitate this, greater knowledge of the responsiveness of these tumours to different treatment strategies is required, both through clinical and preclinical studies (10).

Previous in vitro studies have investigated response to ionizing irradiation (IR) in a range of HNSCC cell lines. These studies describe differences in sensitivity to IR correlating with HPV status (11-17). Some of these reports 
suggest an association between a greater intrinsic cellular radio-sensitivity and increased survival in $\mathrm{HPV}^{+} \mathrm{HNSCC}$ cell lines (11-13). However, in these studies the numbers of $\mathrm{HPV}^{+}$ OPSCC cell lines have been limited, making it difficult to assess the generalizability of these observations (11-17). There is a clear need to assess the correlation between HPV status and radiosensitivity in a greater number of cell lines, but $\mathrm{HPV}^{+}$ OPSCC cell lines are relatively scarce and are notoriously difficult to establish.

In 2014, to our knowledge, there are eight published $\mathrm{HPV}^{+} \mathrm{HNSCC}$ cell lines: UPCI-SCC-90, UPCI-SCC-154, UPCI-SCC-152, UM-SCC-104, UM-SCC-47, 93-VU-147T, UD-SCC-2 and UT-SCC-45 (11-18). In 2018, we established and characterised two new $\mathrm{HPV}^{+}$cell lines CU-OP-2 and CU-OP-3 (18). In this report, two additional cell lines CU-OP-17 and CU-OP-20 have been derived and characterised. We have used these 4 novel lines, and 6 established OPSCC lines to investigate the effects of IR on surviving fraction (SF), cell cycle distribution, and global transcription patterns, similar to studies performed by others (11-13). In addition, circos plots were established, to identify possible changes in HPV integration sites.

\section{Materials and methods}

Patients, cell lines, and sample collection. Five $\mathrm{HPV}^{+}$and five $\mathrm{HPV}^{-}$OPSCC cell lines were included in this study.

To derive novel lines, TSCC biopsies were obtained, during the diagnostic procedure (where ultimately diagnosis is finalised by multidisciplinary meetings) from patients, prior to treatment at Cardiff and Vale University Health Board, in concordance with Ethical and NHS R\&D approval (reference number 13/WA/0002), by written consent. The derivation of $\mathrm{HPV}^{+} \mathrm{CU}-\mathrm{OP}-2$ and CU-OP-3 has been described previously (18). CU-OP-17 and CU-OP-20, described here for the first time, were established using the same processes (18), and were shown below to be $\mathrm{HPV}^{-}$and $\mathrm{HPV}^{+}$respectively. The identity of all CU-OP cell lines included in this report was established by short tandem repeats (STR) as described previously for CU-OP-2 and CU-OP-3 (18), and by Public Health England (Promega, Southampton, UK) for CU-OP-17 and CU-OP-20. The characteristics of the patients from which CU-OP-17 and CU-OP-20 were derived and their tumours are shown in Table I. For CU-OP-2 and CU-OP-3 these details have been published previously (18). All CU-OP cell lines were grown on 60 Gy irradiated J2 3T3 feeder layers (a kind gift from Dr Sally Roberts, University of Birmingham, UK) in GMEM medium with $10 \%$ FBS, and further details have been described previously (18).

$\mathrm{HPV}^{+} \mathrm{UM}^{-S C C}-47$ and HPV${ }^{-}$UM-SCC-6, UM-SCC-19, UM-SCC-74a and UM-SCC-4 were obtained from Professor Thomas Carey at the University of Michigan USA. HPV UPCI-SCC-90 was purchased from Deutsche Sammlung von Mikoorganismen und Zellkulturen (DSMZ), Leibniz, Germany. All these cell lines are described in the data base https://web.expasy.org/cellosaurus/. These cell lines were all cultured in DMEM (Sigma-Aldrich; Merck KGaA), with $1 \% \mathrm{~L}$-glutamine, $100 \mathrm{U} / \mathrm{ml}$ of penicillin, and $100 \mu \mathrm{g} / \mathrm{ml}$ streptomycin and $10 \%$ foetal bovine serum (Sigma-Aldrich; Merck KGaA).
Human epithelial keratinocytes (HEKn) were purchased from Thermo Fisher Scientific, Inc. and grown as described previously in EpiLife media (18).

All cell lines were tested for absence of mycoplasma, by standardised PCR, using the Venor ${ }^{\circledR} \mathrm{GeM}$ detection kit (Minerva Biolabs), which is specific to the highly conserved 16s rRNA coding region, thereby detecting a wide range of mycoplasma species.

HPV PCR. The presence of HPV in CU-OP-17 and CU-OP-20 was confirmed by a bead-based multiplex assay for $27 \mathrm{HPV}$ types as described in detail previously (19). This PCR uses $\mathrm{BSG}^{+} / \mathrm{G}^{+}$primers targeting the $\mathrm{L} 1$ region, as well as primers targeting the HPV16 E6 region, and includes the analysis of all high-risk HPV types using a PCR-based bead-based multiplex-assay on a MagPix instrument (Luminex Corp.) as previously described (19). A mean fluorescence intensity (MFI) value above $1.5 \mathrm{x}$ background +8 for specific HPV primers were considered as HPV-positive as previously described (19).

Clonogenic assay and analysis. Cells were cultured at low cell densities, more specifically in ranges of 2,500-12,500 cells per plate on 6-cm culture dishes (VWR) and incubated at $37^{\circ} \mathrm{C}$ with $5 \% \mathrm{CO}_{2}$ for $24 \mathrm{~h}$. Cell density per cell line, was defined before the initiation of clonogenic assays. Cell density, was defined by individual cell line density tests (using ranges of cells between 1,000-20,000 cells/well lasting for $\sim 10-15$ days depending on the cell line. After $24 \mathrm{~h}$ the cells were irradiated with 0-6 Gy (Gammacell-1000 MDS Nordion; a caesium-137 source) and the media were changed after 7 days. The assays were stopped 10-15 days later (depending on the growth of the cell lines) and the cells stained with crystal violet, and the colonies counted using a Colony counter (ColonyDoc-It Imaging Station, UVP).

Data analysis and statistical evaluation of clonogenic assay. All experiments were performed in triplicates. The plating efficiency (PE) and survival fraction (SF) of each cell line per IR dose was calculated. Cells were classified as radiosensitive $\mathrm{SF}<0.40$ or radio-resistant $\mathrm{SF}>0.40$ as described before (20). The mean value was calculated and standard deviations (SD) are indicated as error bars. Statistical evaluation was performed using GraphPad Prism (GraphPad Version 7, GraphPad Software), by using a two-way ANOVA with a Sidak post-test. Strength of significance is indicated as follows: ${ }^{*} \mathrm{P}<0.05,{ }^{* *} \mathrm{P}<0.01,{ }^{* * *} \mathrm{P}<0.01$ and $\left.{ }^{* * * *} \mathrm{P}<0.001\right)$. ns stands for not significant.

\section{Flow cytometry}

Sample preparation and collection. Cells were cultured without feeder cells in 6-cm culture dishes (VWR) and treated with 0-6 Gy 24 h after seeding. Untreated cells were collected $24 \mathrm{~h}$ after seeding, while irradiated cells were collected 8, 24 and $48 \mathrm{~h}$ after treatment. Approximately 500,000 cells/cell line were fixed in $1 \mathrm{ml}$ of $70 \%$ ethanol in fluorescence-activated cell sorting (FACS) tubes and stored at $-20^{\circ} \mathrm{C}$ for at least $1 \mathrm{~h}$. Cells were then washed with PBS, incubated with $100 \mu 1$ of RNase A (10 $\mu \mathrm{g} / \mathrm{ml}$ ) (Sigma-Aldrich; Merck KGaA) for $45 \mathrm{~min}$ at $37^{\circ} \mathrm{C}$, centrifuged at $270 \mathrm{x} \mathrm{g}$, and resuspended in $200 \mu \mathrm{l}$ propidium iodide (PI) solution (50 $\mu \mathrm{g} / \mathrm{ml})$ (Sigma-Aldrich; Merck KGaA) and incubated for $15 \mathrm{~min}$ at $37^{\circ} \mathrm{C}$. 
Cell cycle measurement and statistical analysis. The PI-stained cells were analysed using a BD Accuri C6 (BD Biosciences) low-pressure flow cytometer (absorbance $488 \mathrm{~nm}$ ). Data were extracted as FCS files and the cell cycle distribution was analysed with FlowJo analysis software (version 10; FlowJo LLC), using the cell cycle tool, based on the Watson pragmatic algorithm (21). For each cell cycle phase the mean value was calculated based on a total of three experimental runs. Each IR dose and time point were compared to the non-treated sample 'time zero', to quantify changes in cell cycle distribution after IR treatments. Excel 2016 was used to generate $100 \%$ stacked bar charts. Statistical analysis was performed in GraphPad Prism 7 (GraphPad Software, Inc.) using a two-way ANOVA with a Sidak post-test to the panel of cell lines (treatment dose and time points). Strength of significance is indicated as follows: ${ }^{*} \mathrm{P}<0.05,{ }^{* * *} \mathrm{P}<0.01,{ }^{* * *} \mathrm{P}<0.01$ and $\left.{ }^{* * * *} \mathrm{P}<0.001\right)$. ns stands for not significant.

mRNA sequencing

Experimental set up. Cells (untreated and $2 \mathrm{~Gy}$ ) were collected $24 \mathrm{~h}$ after treatment and RNA was extracted with QiaAMP Mini kit (Qiagen) according to the manufacturer's instructions.

Library preparation. Library preparation and validation for mRNA sequencing was performed through a commercial service/collaboration with Wales Gene Park (Cardiff University, UK). Library preparation, including depletion of ribosomal RNA was carried out using the Illumina ${ }^{\circledR}$ TruSeq $^{\circledR}$ Stranded Total RNA with Ribo-Zero Gold ${ }^{\mathrm{TM}}$ kit (Illumina Inc.) according to the instructions of the manufacturer.

Sequencing and data analysis. A 75-base paired-end dual index read format was used on the Illumina ${ }^{\circledR}$ HiSeq2500 in high-output mode by the Wales Gene Park (Cardiff University, UK). Sequencing data were analysed by the bioinformatics service by Dr Peter Giles at the Wales Gene Park (Cardiff University, UK). Trimmed reads were mapped against a combined human sequence genome hg19 and an HPV16 genome reference sequence NC_001526 using STAR (Alex Dobin, Git Hub) to generate circos plots. For both exons and transcripts, gene expression counts were calculated, using Subread feature Counts Version 1.5.1 (22). Data were visualised using The Integrative Genomics Viewer (IGV) downloaded from the Broad Institute (23) and Geneview software (written by Dr Peter Giles of the Wales Gene Park).

Differential gene expression. The DEseq2 analysis tool was used to identify differentially expressed genes (statistical analysis of count matrices for systematic changes between conditions) (24). For multiple testing and false discovery issues, the generated $\mathrm{P}$-values were corrected using the FDR method (25). The data are shown in heatmaps in 3 colour patterns, where green represents underexpressed (compared to median); black represents values approximately median expression; and red represents overexpressed values.

Human viral fusion transcript plots (circos plots). Circos plots were used to visualise and identify human (hg19) and viral (HPV-16) mRNA fusion transcripts (26). 


\section{Results}

Derivation of novel OPSSC cell lines $C U-O P-17$ and CU-OP-20. Explant cultures using fresh primary biopsies from two patients with TSCC were attempted as described previously (18). In addition, and the identity of the cell lines CU-OP-17 and CU-OP-20 to the tumour biopsies was confirmed by STR. Details of these two patients and their tumours are shown in Table I. The HPV status of the biopsy and resulting cell line was assessed by PCR-based bead-based multiplex-assay on a MagPix instrument and the obtained MFIs calculated as described above: CU-OP-20 tested positive for HPV-16; CU-OP-17 was HPV TP53 status assessed by RNA sequencing was considered as being wild-type in both cell lines.

\section{Surviving fraction $(\mathrm{SF})$ of $\mathrm{HPV}^{+}$and $\mathrm{HPV}$}

OPSCC cell lines after ionising irradiation (IR). The sensitivity of the panel of 10 OPSCC cell lines to IR was assessed using clonogenic survival assays. The SFs of all OPSCC cell lines to $0.5-6$ Gy respectively are shown in Fig. 1, and Tables II and III, respectively.

Special focus was put on an indicated clinically relevant dose of $2 \mathrm{~Gy}$ (20), which identified three $\mathrm{HPV}^{+}$OPSCC cell lines UM-SCC-47 $(\mathrm{SF}=14.1)$ UPCI-SCC-90 $(\mathrm{SF}=28.3)$ and CU-OP-20 (SF=20.4) and only one HPV ${ }^{-}$OPSCC cell line UM-SSC-19 $(\mathrm{SF}=32.4)$ as radiosensitive, Tables II and III, respectively. All other cell lines were by definition radioresistant (20). Furthermore, for $2 \mathrm{~Gy}, \mathrm{HPV}^{+} \mathrm{OPSCC}$ cell lines tended to be more sensitive and showed a wider variability in SF values (14.1-60.4, mean 33.6) than the HPV- OPSCC cell lines (32.4-71.8, 51.4) $\mathrm{P}=0.0754$ (Mann-Whitney one-tailed $\mathrm{t}$-test as conducted in (13) (Fig. 1, Tables II and III, respectively).

There were significant differences in SF within the $\mathrm{HPV}^{+}$ and HPV groups after IR with $2 \mathrm{~Gy}$. $\mathrm{HPV}^{+} \mathrm{UM}-\mathrm{SCC}-47$ and CU-OP-20 were more radiosensitive to 2 Gy compared to CU-OP-2 and CU-OP-3, and UPCI-SSC-90 was more radiosensitive than CU-OP-3 (for all at least $\mathrm{P}<0.05$ ) (Fig. 1A). Among the HPV- OPSCC cell lines, UM-SCC-19 was more radiosensitive than CU-OP-17 (P<0.0001) (Fig. 1B). Fig. 1C shows the SF values in more detail after treatment with $2 \mathrm{~Gy}$ for all OPSCC cell lines as well as HEKn. Fig. 1C demonstrates that $\mathrm{HPV}^{+}$OPSCC cell lines have a variable sensitivity to IR, as well as an evident overlap with HPV OPSCC cell lines, but still tend to be more radiosensitive than the HPV- OPSCC cell lines.

Effects of IR on cell cycle distribution in $\mathrm{HPV}^{+}$and $\mathrm{HPV}$ OPSCC cell lines. To gain insight into the mechanisms underlying the differences in sensitivity to IR, the cell lines were treated with IR and the effects on cell cycle distribution were assessed by flow cytometry. IR induced cell cycle effects primarily on $\mathrm{HPV}^{+}$OPSCC as compared to $\mathrm{HPV}^{-} \mathrm{OPSCC}$ and mainly in the proportion of cells in $\mathrm{G} 2$, and the data are presented in detail below.

Changes in $\mathrm{G} 2$ arrest in $\mathrm{HPV}^{+}$OPSCC cell lines. No significant changes were observed in cell cycle distribution $8 \mathrm{~h}$ after 2-6 Gy for any cell line. However, after 24 h (2 Gy), a significant increase in cells in $\mathrm{G} 2$ phase was observed for two $\mathrm{HPV}^{+}$OPSCC cell lines: UM-SCC-47 (the most radiosensitive line, $\mathrm{P}=0.0135$ ) and $\mathrm{CU}-\mathrm{OP}-3$ (the most radioresistant line,
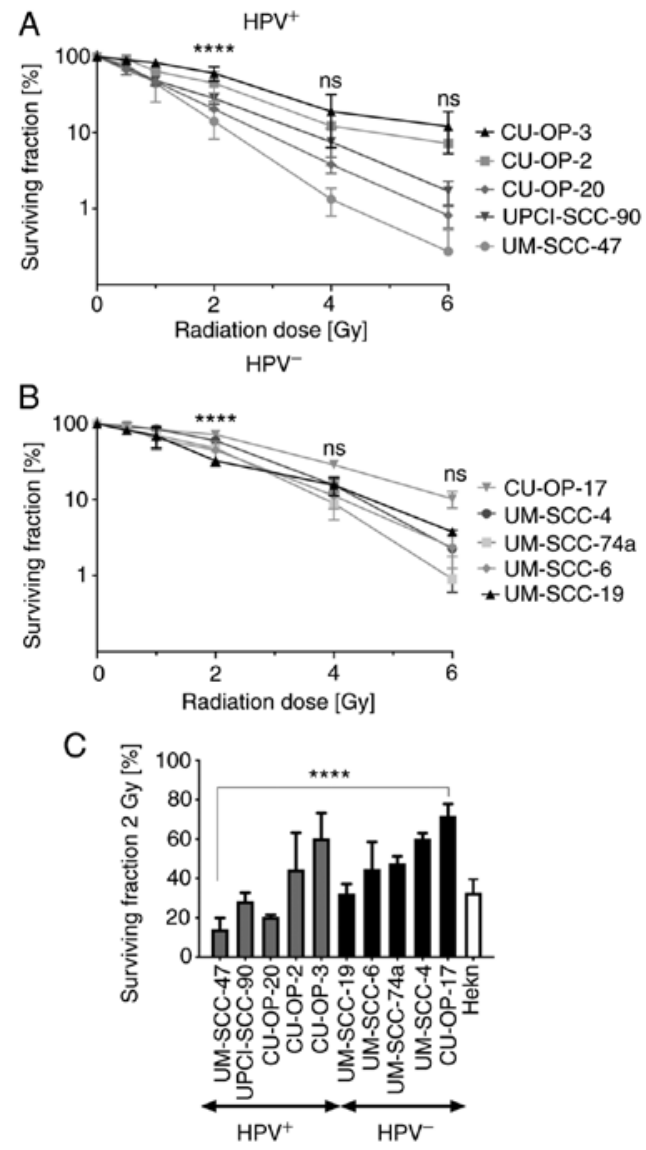

Figure 1. Surviving fraction (SF) of $\mathrm{HPV}^{+}$and $\mathrm{HPV}^{-}$cell lines following ionizing irradiation. (A) SF of $\mathrm{HPV}^{+}$cell lines. At $2 \mathrm{~Gy}$ the difference in survival between the most radiosensitive (UM-SCC-47) and the most radioresistant $(\mathrm{CU}-\mathrm{OP}-3)$ cell lines was significant $\left({ }^{* * * *} \mathrm{P}<0.0001\right)$. Additional significant differences are noted only in the text of the results section. ns, not significant. Error bars indicate standard deviations. (B) SF of HPV cell lines to IR. At $2 \mathrm{~Gy}$ the difference in survival between the most radiosensitive (UM-SCC-19) and the most radioresistant (CU-OP-17) cell lines was significant $\left(^{* * * * *} \mathrm{P}<0.0001\right)$. Additional significant differences are noted only in the text of the results section. ns, not significant. Error bars indicate standard deviations. (C) SF after treatment with $2 \mathrm{~Gy}$ for all $\mathrm{HPV}^{+}$and $\mathrm{HPV}^{-} \mathrm{SCC}$ cell lines and the human epithelial keratinocyte cell line HEKn. As example of statistical significance, the comparison of UM-SCC-47 to CU-OP-17 is presented $\left({ }^{* * * *} \mathrm{P}<0.0001\right)$. For this specific comparison a one-way ANOVA with a Sidak post-test was used. HPV, human papillomavirus.

$\mathrm{P}=0.0075$ ) (Fig. $2 \mathrm{~A}$ and $\mathrm{E}$ ). For the $6 \mathrm{~Gy}$ treatment after $24 \mathrm{~h}$, all $\mathrm{HPV}^{+} \mathrm{OPSCC}$ cell lines showed a significant increase in G2 arrest (UM-SCC-47, P<0.0001; UPCI-SCC-90, P<0.0001; CU-OP-20, P=0.0004; CU-OP-2, P=0.0019; and CU-OP-3, $\mathrm{P}<0.0001$ ) (Fig. 2). After $48 \mathrm{~h}$ post $6 \mathrm{~Gy}$ treatment the proportion of cells in G2 was generally reduced. In UM-SCC-47, UPCI-SSC-90 and CU-OP-2, the reduction in G2 fraction was significant $(\mathrm{P}<0.05)$ (Fig. 2A-C).

\section{Changes in $\mathrm{G} 2$ arrest in $\mathrm{HPV}$}

OPSCC cell lines. Compared to untreated controls, at $2 \mathrm{~Gy}$ after $24 \mathrm{~h}$, a significant increase in cells in $\mathrm{G} 2$ was observed in one HPV OPSCC cell line, UM-SCC-4, $\mathrm{P}=0.0222$ (Fig. 3C). With 6 Gy after $24 \mathrm{~h}$, an increase in G2 arrest was only observed in two lines, and these were the two most IR resistant ones of the five HPV cell lines: UM-SCC- $4, \mathrm{P}=0.0027$ and CU-OP-17, $\mathrm{P}=0.0052$ (Fig. $3 \mathrm{C}$ and E). It was notable that in 
Table II. Surviving fraction (SF) of $\mathrm{HPV}^{+}$cell lines after treatment with 0.5-6 Gy.

\begin{tabular}{|c|c|c|c|c|c|}
\hline \multirow[b]{3}{*}{ Treatment dose (Gy) } & \multicolumn{5}{|c|}{ Surviving fraction $(\%)$} \\
\hline & \multicolumn{3}{|c|}{$\mathrm{HPV}^{+}$cell lines sensitive to IR } & \multicolumn{2}{|c|}{$\begin{array}{c}\mathrm{HPV}^{+} \text {cell } \\
\text { lines resistant to IR }\end{array}$} \\
\hline & UMSCC-47 & UPCI-SCC-90 & CU-OP-20 & CU-OP-2 & CU-OP-3 \\
\hline 0.5 & 78.4 & 72.5 & 66.8 & 93.6 & 90.5 \\
\hline 1 & 44.4 & 48.5 & 46.9 & 64.1 & 83.3 \\
\hline $2^{\mathrm{a}}$ & 14.1 & 28.3 & 20.4 & 44.7 & 60.4 \\
\hline 4 & 1.3 & 7.5 & 3.8 & 12.2 & 19.1 \\
\hline 6 & 0.3 & 1.7 & 0.8 & 7.2 & 12.1 \\
\hline
\end{tabular}

${ }^{\text {a SF }}<40 \%$ defined as sensitive to IR according to (20). IR, ionizing irradiation; HPV, human papillomavirus.

Table III. Surviving fraction (SF) of HPV- OPSCC cell lines and HEKn after treatment with 0.5-6 Gy.

\begin{tabular}{|c|c|c|c|c|c|c|}
\hline \multirow[b]{3}{*}{ Treatment dose (Gy) } & \multicolumn{5}{|c|}{ Surviving fraction $(\%)$} & \multirow{3}{*}{$\frac{\begin{array}{c}\text { Non-cancer cell } \\
\text { line response to IR }\end{array}}{\text { HEKn }}$} \\
\hline & \multirow{2}{*}{$\begin{array}{l}\mathrm{HPV}^{-} \text {cell line } \\
\text { sensitive to IR } \\
\text { UMSCC-19 }\end{array}$} & \multicolumn{4}{|c|}{$\mathrm{HPV}^{-}$cell lines resistant to IR } & \\
\hline & & UMSCC-6 & UMSCC-74a & UMSCC-4 & CU-OP-17 & \\
\hline 0.5 & 81.7 & 87.4 & 85.3 & 92.3 & 94.3 & 79.8 \\
\hline 1 & 69.1 & 62.9 & 70.1 & 85.1 & 84.2 & 70.6 \\
\hline $2^{\mathrm{a}}$ & 32.4 & 44.9 & 47.7 & 60.1 & 71.8 & 32.7 \\
\hline 4 & 15.5 & 11.3 & 8.9 & 15.6 & 28.8 & 17.6 \\
\hline 6 & 3.8 & 2.4 & 0.9 & 2.3 & 10.3 & 1.6 \\
\hline
\end{tabular}

${ }^{\text {aSF }},<40 \%$ defined as sensitive to IR according to (20).

contrast to the $\mathrm{HPV}^{+}$lines, neither UM-SCC-4, nor CU-OP-17 showed a reduction in $\mathrm{G} 2$ fraction after $48 \mathrm{~h}$.

\section{Changes in G1 in $\mathrm{HPV}^{+}$and $\mathrm{HPV}^{-}$}

OPSCC cell lines. Roughly, 50-60\% of $\mathrm{HPV}^{+}$and HPV- OPSCC cells were initially in G1, and few changes occurred 8-48 h after IR (Figs. 2 and 3). Compared to controls, with 2 Gy after $8 \mathrm{~h}$, decreases in the proportion of cells in G1 were noted in $\mathrm{HPV}^{+}$UM-SCC-47, P=0.0031; HPV ${ }^{-} \mathrm{UM}-\mathrm{SCC}-4, \mathrm{P}<0.0001$; and HPV UM-SCC-74a, P=0.0168; (Figs. 2A and 3C and D), and this was the case after $24 \mathrm{~h}$ for CU-OP-17, $\mathrm{P}=0.0402$; (Fig. 3E), while instead an increase in G1 was seen at $24 \mathrm{~h}$ in UM-SCC-74a, P=0.0037; (Fig. 3D). At $6 \mathrm{~Gy}$, after $24 \mathrm{~h}$, a decrease in $\mathrm{G} 1$ could be seen for $\mathrm{HPV}^{+} \mathrm{UM}-\mathrm{SCC}-47$, $\mathrm{P}<0.0001$; and UPCI-SCC-90, $\mathrm{P}<0.0001$; (Fig. 2A and B) and $\mathrm{HPV}^{-}$UM-SCC-19, $\mathrm{P}=0.0312$; and UM-SCC-4, $\mathrm{P}=0.0305$ (Fig. 3A and C).

\section{Changes in $\mathrm{S}$-phase in $\mathrm{HPV}^{+}$and $\mathrm{HPV}^{-}$}

OPSCC cell lines. Compared to untreated controls some changes in the proportion of cells in S phase occurred early $(8 \mathrm{~h})$, but not later (24-48 h) after IR in both $\mathrm{HPV}^{+}$and $\mathrm{HPV}^{-}$ OPSCC cell lines (Figs. 2 and 3). Compared to controls, with
2 Gy after $8 \mathrm{~h}$ an increase in the proportion of cells in S-phase was observed in $\mathrm{HPV}^{+} \mathrm{UM}-\mathrm{SCC}-47$; $\mathrm{P}=0.0002$; (Fig. 2A) and $\mathrm{HPV}^{-}$UM-SCC-4, $\mathrm{P}<0.0001$; (Fig. 3C). Compared to controls, with 6 Gy after 8 h, HPV ${ }^{+} \mathrm{UM}-\mathrm{SCC}-47, \mathrm{P}=0.0004$; UPCI-SCC-90, P=0.0123; CU-OP-2; P=0.0144; (Fig. 2A-C) and $\mathrm{HPV}^{-} \mathrm{UM}-\mathrm{SCC}-4, \mathrm{P}=0.0061$; (Fig. 3C) showed increases in the proportion of cells in S-phase.

No changes in the cell cycle after IR of HEKn. No significant changes were observed in the cell cycle of HEKn between the non-treated samples and the 2 Gy and 6 Gy samples $(8,24$ and 48 h) (Fig. S1). One representative flow cytometry plot of each cell line is presented in Figs. S2 and S3.

Influence of IR on HPV integration. RNA sequencing was used to identify the presence on human: Viral fusion transcripts before and after treatment with 2 Gy IR. This data was visualised using circos plots, which demonstrated fusion transcripts as described previously for UM-SCC-47, UPCI-SCC-90, CU-OP-2 and CU-OP-3 (18). The main integration sites were: UM-SCC-47-chromosome 3 to TP63 from E6/E7; UPCI-SCC-90-C9orf156 (chromosome 9 open reading frame 156 and 200 bp before FOXE1 

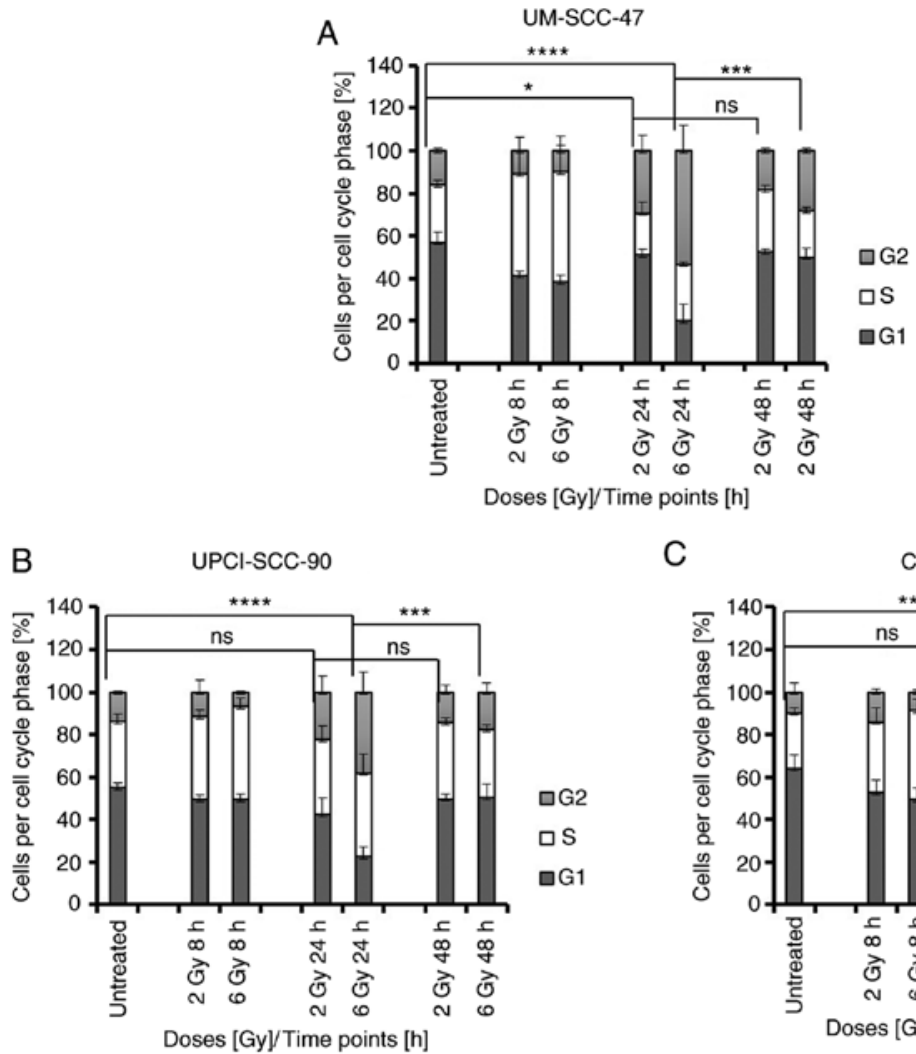

C

CU-OP-2
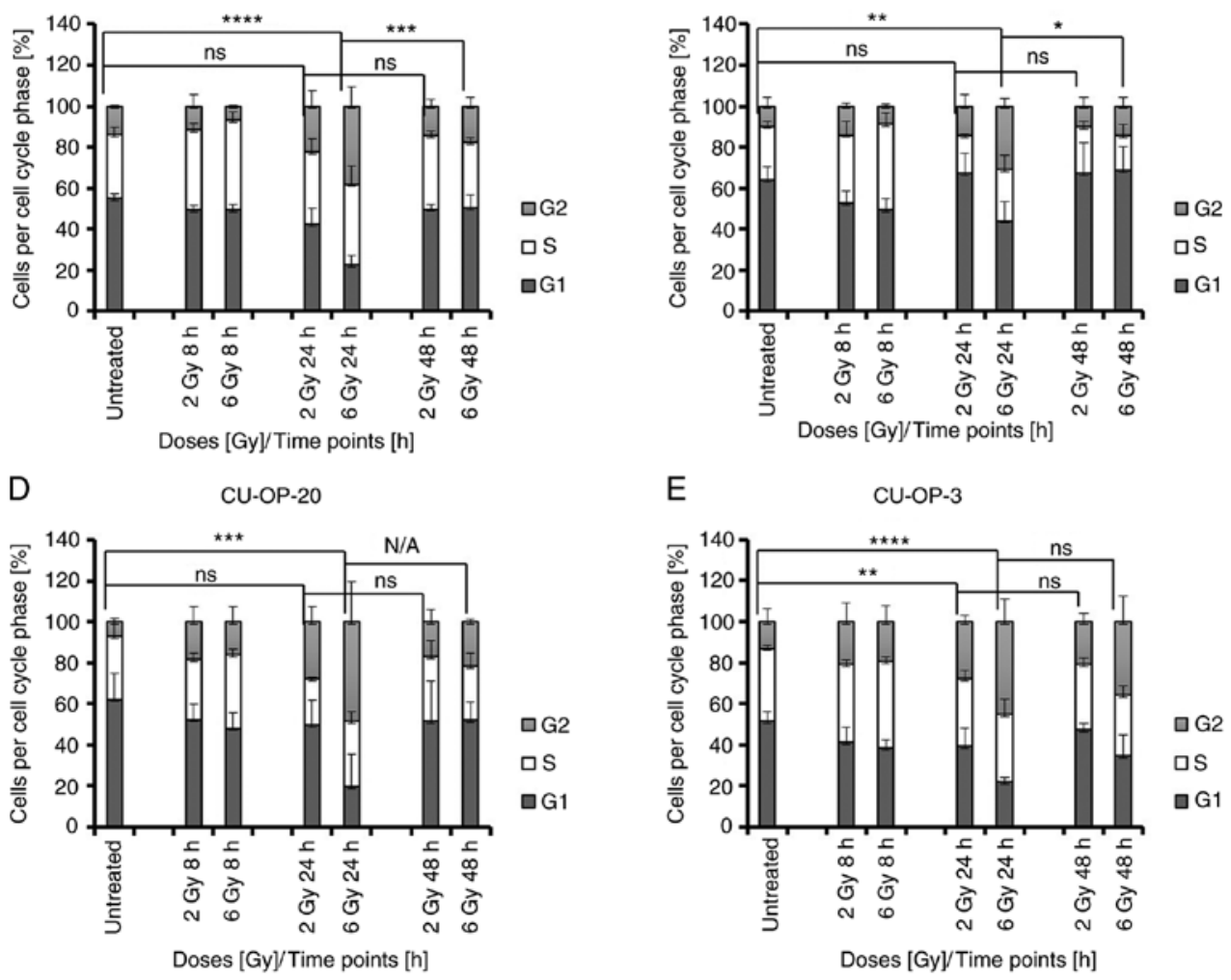

Figure 2. Distribution of cells in G1, S-phase and G2 at different time points after ionizing irradiation (2 and 6 Gy) for HPV+ cell lines: (A) UM-SCC-47, (B) UPCI-SCC-90, (C) CU-OP-2, (D) CU-OP-20 and (E) CU-OP-3. All indicated statistical significance values in the figure apply only to cells in G2 phase. The statistics present visually the significance between the untreated G2 phase and the treated G2 phases at the presented time points $\left({ }^{*} \mathrm{P}<0.05,{ }^{* *} \mathrm{P}<0.01\right.$, ${ }^{* * *} \mathrm{P}<0.01$ and $\left.^{* * * *} \mathrm{P}<0.001\right)$. Untreated samples presented represent time zero. N/A, not available; ns, not signifcant. All other statistical differences for G1 and $\mathrm{S}$ are presented in the text of the results section. HPV, human papillomavirus.

from E7; CU-OP-2-chromosome 10 to E2 (YME1L1 gene at introns 6 and 7); CU-OP-3-chromosome 20 at the gene CEBPB (CCAT/enhancer binding protein $\beta$ ) (Fig. S4) (18). Novel minor HPV integration sites were identified for CU-OP-20, but a high incidence of fusion transcripts above 5 mapped reads, as described previously (18) were not disclosed (Fig. S4). Treatment with 2 Gy did not change the main integration sites of UM-SCC-47, UPCI-SCC-90, CU-OP-2 and CU-OP-3 (Fig. S4). However, the frequency of additional minor chromosomal integration sites per chromosome increased after 2 Gy IR, i.e. for the radiosensitive lines: UM-SCC-47, from 3 to 16 ; UPCI-SCC-90, from 17 to 20; and CU-OP-20; from 7 to 20; as well as for the radioresistant lines: CU-OP-3, from 6 to 21 and CU-OP-2, from 1 to 10 (Fig. S4A and B, respectively).
Changes in gene expression in $\mathrm{HPV}^{+}$and $\mathrm{HPV}^{-}$

OPSCC cell lines after IR with 2 Gy. The RNA-sequencing data was also examined to determine whether there were consistent differences in response to IR between the $\mathrm{HPV}^{+}$and $\mathrm{HPV}^{-}$cell lines. An unsupervised analysis comparing treated and untreated cells was initially performed. After correction for multiple testing, 519 transcripts or genes were indicated as significant (data not shown). This indicated 2 Gy had a significant effect on transcription of multiple genes. The top 19 genes with significant differences after treatment of the OPSCC lines with $2 \mathrm{~Gy}$ are presented in Table SI. None of the genes however, showed an obvious link to DNA repair, DNA damage or stress mechanisms, while transcription of the HPV oncogenes, E6 and E7, was noted in all $\mathrm{HPV}^{+}$cell lines (data not shown). 

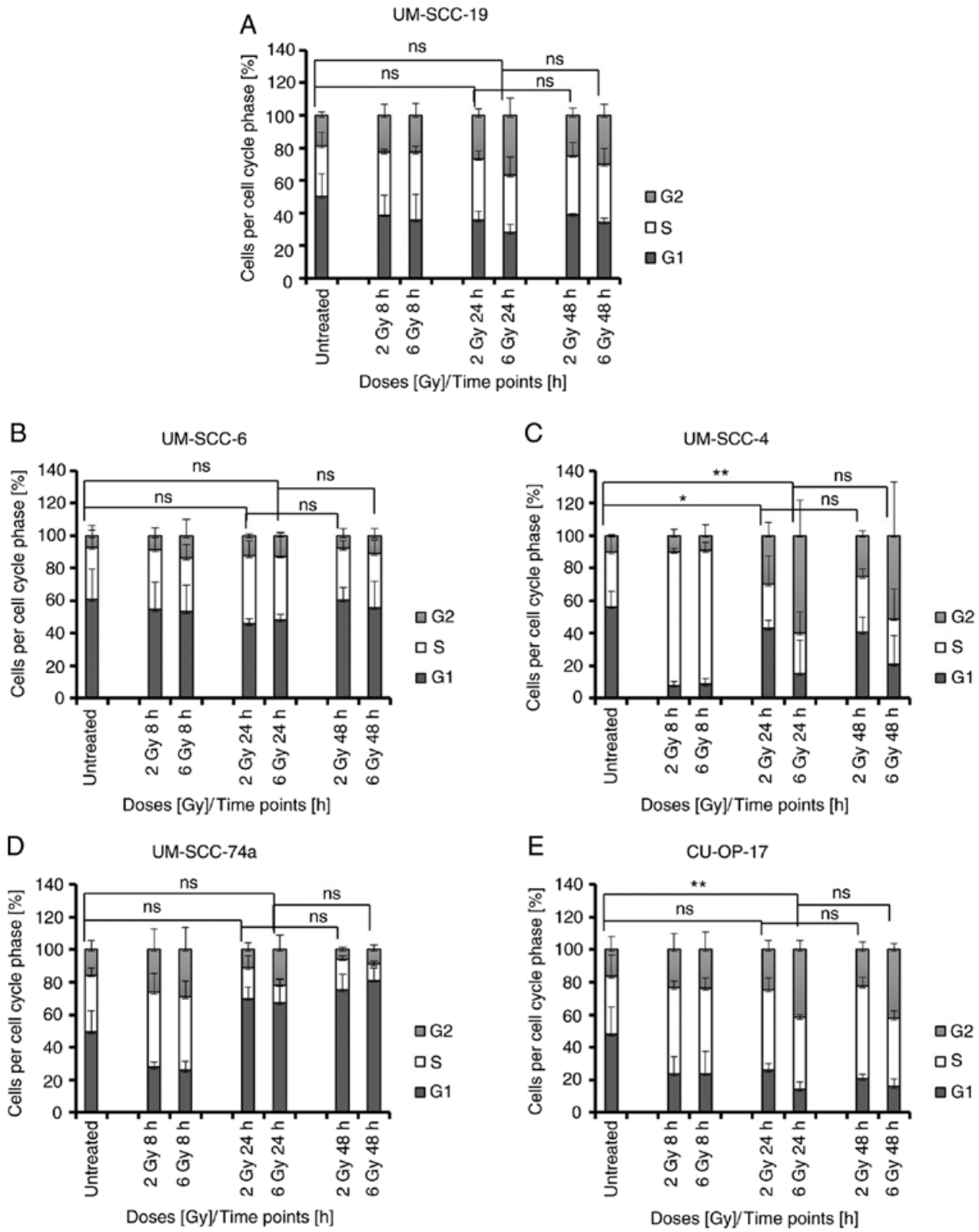

Figure 3. Distribution of cells in the G1, S-phase and G2 at different time points after ionizing irradiation of HPV-cell lines with 2 and 6 Gy: (A) UM-SCC-19, (B) UM-SCC-6, (C) UM-SCC-4, (D) UM-SSC-74a and (E) CU-OP-17. All indicated statistical significance values in the figure apply only to cells in G2 phase. The statistics present visually the significance between the untreated G2 phase and the treated G2 phases at the presented time points $\left({ }^{*} \mathrm{P}<0.05\right.$ and $\left.{ }^{* *} \mathrm{P}<0.01\right)$. Untreated samples presented represent time zero. ns, not signifcant. All other statistical differences for G1 and S are presented in the text of the results section. HPV, human papillomavirus.

\section{Discussion}

The present study investigated responses to ionising irradiation (IR) in five human papillomavirus-positive $\left(\mathrm{HPV}^{+}\right)$and five $\mathrm{HPV}^{-}$oropharyngeal squamous cell carcinoma (OPSCC) cell lines. Clonogenic survival assays, flow cytometry and RNA sequencing were used to assess survival, cell cycle distribution and HPV integration. We also report derivation and characterisation of two novel OPSCC cell lines. $\mathrm{HPV}^{+}$OPSCC cell lines showed greater variation in radiosensitivity than was apparent among the $\mathrm{HPV}^{-}$cell lines, and we observed a tendency for greater sensitivity to IR in $\mathrm{HPV}^{+}$lines, although the correlation between radiosensitivity and HPV status was not perfect. $\mathrm{HPV}^{+}$ cells more frequently demonstrated an increase in G2 arrest following IR as compared to the HPV OPSCC cell lines. It was interesting and potentially clinically relevant to note that radiation treatment resulted in a marked increase in novel HPV: Human fusion transcripts, which may suggest that radiation could facilitate integration of HPV DNA into novel genomic sites. However, due to the limited number of available cell lines and without further studies, a definite conclusion cannot be made after the current study. RNA sequencing did not indicate major changes in transcription of genes associated with DNA repair, DNA damage or stress mechanisms.

Following irradiation of $\mathrm{HPV}^{+}$cell lines (UM-SCC-47, UPCI-SCC-90, CU-OP-2, CU-OP-3, and CU-OP-20) and the HPV cell lines (UM-SCC-6, UM-SCC-4, UM-SCC-19, UM-SCC-74a and CU-OP-17), our observations show some 
important differences from earlier reports (11-13). These data are based on an expanded panel of cell lines, since three more $\mathrm{HPV}^{+}$and two $\mathrm{HPV}^{-}$OPSCC cell lines $\left(\mathrm{HPV}^{+} \mathrm{CU}-\mathrm{OP}-2\right.$, CU-OP-3, CU-OP-20 and HPV CU-OP-17 and UM-SCC-74a) were added to the previously relatively limited panel of IR tested OPSCC cell lines (11-18). Notably, in this report we did not find that $\mathrm{HPV}^{+} \mathrm{OPSCC}$ cell lines were consistently and significantly more sensitive to IR relative to $\mathrm{HPV}^{-}$OPSCC cells (11-13), although this trend was evident in a limited number of lines. We suggest that it would be more accurate to state that $\mathrm{HPV}^{+}$ OPSCC cell lines show wider variation in radio-sensitivity than is observed among the HPV- OPSCC cell lines.

The wider variability in sensitivity to IR in the $\mathrm{HPV}^{+}$group is in line with one previous report, where the authors also therefore suggest caution in de-intensification of therapy via dose reduction (13). An example of this in the present study was that the $\mathrm{HPV}^{+} \mathrm{CU}-\mathrm{OP}-2$ and CU-OP-3 cell lines were relatively radioresistant as compared to the other $\mathrm{HPV}^{+}$cell lines.

Of note, the $\mathrm{HPV}^{+} \mathrm{CU}-\mathrm{OP}-20$, which according to our circos plots lacked major integrated HPV sites, was among the most radiosensitive $\mathrm{HPV}^{+}$OPSCC cell line. The data are of course very limited, but would be consistent with suggestions that $\mathrm{HPV}^{+}$OPSCC with episomal HPV DNA may have an even better prognosis $(27,28)$.

Similar to other publications, we found that upon IR the proportion of $\mathrm{HPV}^{+}$cell lines often exhibited an increase in $\mathrm{G} 2$ arrest as compared to HPV- OPSCC lines, while changes in the proportion of cells in G1 and S-phase were not as apparent for any of the OPSCC cell lines (11-13). In two of the previous studies it was hypothesised that due to HPV16 E6-mediated degradation of p53, a greater G2 arrest should be expected in the $\mathrm{HPV}^{+}$OPSCC cell lines $(12,13)$. Notably, an increase in G2 arrest was the case for the $\mathrm{HPV}^{+}$OPSCC cell lines in this study, irrespective as to whether the cell lines were radiosensitive or radioresistant. The data would still be in line with the hypothesis above indicating that it is due to HPV16 E6-mediated degradation of p53 rather than a correlation to radiosensitivity per se $(12,13)$. This would be consistent with the observation that the most radiosensitive line, UM-SCC-47, and the most radioresistant CU-OP-3 were the two cell lines with the highest proportion of G2 arrest. To our knowledge this has not been demonstrated before. In fact, in an earlier report the numbers of cells arrested in G2 correlated to the radiosensitivity of the $\mathrm{HPV}^{+}$OPSCC cell lines (13). The differences in the data reported between the present study and the study by Rieckmann et al (13) reflects the difficulties in drawing generalised conclusions on limited numbers of $\mathrm{HPV}^{+}$ OPSCC cell lines.

In this study, we also demonstrated an increase in HPV minor integration sites upon IR. We suggest that this could be due to an increase in DNA lesions following IR, and that this promotes integration of HPV DNA (possibly previously episomal) into new genomic locations.

mRNA sequencing IR treated and untreated cells also allowed investigation of changes in transcription of genes correlated to DNA repair, cell cycle arrest and apoptosis. Our data did not indicate an increase in transcription of specific DNA repair genes after IR, which could be interpreted as consistent with the suggestion that the IR sensitivity of $\mathrm{HPV}^{+}$OPSCC cell lines could be due to impaired capability to repair double-stranded DNA breaks (13). However, it may equally reflect the limitation of an experiment involving a single dose at a single time point. Other factors may better explain the better prognosis of $\mathrm{HPV}^{+}$ OPSCC patients. One report suggests e.g. that IR increases the levels of MHC class I in $\mathrm{HPV}^{+}$OPSCC, thereby allowing for better immune recognition, while another study implies the necessity of an immune system to benefit from IR or chemotherapy $(29,30)$. The role of CD47 has also been investigated, showing that this protein is downregulated upon IR, and that this in turn enhances immune recognition of $\mathrm{HPV}^{+}$OPSCC (31).

There are limitations inherent in our study; only 10 OPSCC cell lines were assessed. Nonetheless this is a larger sample than investigated in several previous studies. By including five OPSCC cell lines previously not tested with IR, we could show a tendency, but not a significantly increased sensitivity to IR in $\mathrm{HPV}^{+}$as compared to $\mathrm{HPV}^{-}$OPSCC cell lines, and this may challenge a previous dogma $(12,13)$. Nevertheless, caution is still warranted to draw any major conclusions, since the numbers of cell lines in each category were limited.

Furthermore as already mentioned, another limitation was the special focus on one irradiation dose 2 Gy. However, we could confirm that upon IR, the proportion of cells in G2 increased much more in $\mathrm{HPV}^{+}$OPSCC cell lines as compared to $\mathrm{HPV}^{-}$OPSCC, but in this study the increase in G2 was not correlated to radiosensitivity as reported previously (13). Whether this is due to unique features of the CU-OP-3 cell line, or whether caution should be exercised in regarding G2 arrest as a measure of radiosensitivity, remains to be established.

To summarise, in this report five $\mathrm{HPV}^{+}$and five $\mathrm{HPV}^{-}$ OPSCCs cell lines, including five lines not previously assessed, were examined for radiosensitivity. $\mathrm{HPV}^{+}$OPSCC lines demonstrated a wide range of sensitivity to IR, and importantly not all cell lines were radiosensitive, although they still tended to be more sensitive to irradiation than $\mathrm{HPV}^{-}$OPSCC lines. Furthermore, upon IR, $\mathrm{HPV}^{+}$OPSCCs more often increased the proportion of cells in $\mathrm{G} 2$ arrest as compared to $\mathrm{HPV}^{-}$OPSCC cell lines, but the increases in $\mathrm{G} 2$ arrest were not correlated to radiosensitivity. Lastly, upon treatment with 2 Gy some increases in minor HPV integration sites were noted and changes in gene expression were demonstrated, but not in genes primarily associated with DNA repair.

To conclude, our data suggest that $\mathrm{HPV}^{+}$OPSCC cell lines may possibly vary more in radiosensitivity than previously anticipated, and despite they are generally more sensitive than $\mathrm{HPV}^{-}$OPSCC cell lines, individual variations may exist within both the $\mathrm{HPV}^{+}$and the HPV $\mathrm{HPSCC}^{-}$groups. Furthermore, in spite of the fact that 10 cell lines were tested, the data are limited and additional studies are warranted.

\section{Acknowledgements}

We are very thankful for the support provided by Cancer Research Wales as well as the patients who took part in this study. Tina Dalianis and Nicole Wild are acknowledged for carefully reading the manuscript and providing valuable suggestions, and Ramona Ursu for technical help with the bead based multiplex assay.

\section{Funding}

Grant funding from Cancer Research Wales (grant no. CRW14-506688) supported this study. The funders had no 
role in study design, data collection and analysis, decision to publish, or preparation of the manuscript.

\section{Availability of data and materials}

RNA-seq data discussed in this publication is available in the NCBI's Gene Expression Omnibus (GEO) and is accessible through GEO series accession number GSE153966 (https://www. ncbi.nlm.nih.gov/geo/query/acc.cgi?acc=GSE153966). The remaining datasets used and/or analyzed during the current study are available from the corresponding author on reasonable request.

\section{Authors' contributions}

SH performed the majority of the experiments, interpreted the data, calculated the statistics and contributed to the writing of the manuscript. EP and JJ collaborated with SH and performed some experiments. PG contributed by analyzing and providing the raw data. DO, AAH and ME contributed by identifying and consenting patients and by providing the biopsy samples for the establishment of the cell lines. NP and SM assisted all the authors, with the planning of some experiments, performed combinational analyses, and assisted in final interpretation and presentation of the data. NP and $\mathrm{SH}$ were involved in the work from its initiation and bringing into completion and were involved in writing and finalizing the work and the manuscript. All authors critically read and approved the manuscript.

\section{Ethics approval and consent to participate}

The study with regard to patient material was performed according to permission from the Cardiff and Vale University Health board, in concordance with Ethical and NHS R\&D approval (reference no. 13/WA/0002).

\section{Patient consent for publication}

Not applicable.

\section{Competing interests}

Authors report no competing interests to disclose.

\section{References}

1. Evans M, Newcombe R, Fiander A, Powell J, Rolles M, Thavaraj S, Robinson $\mathrm{M}$ and Powell N: Human papillomavirus-associated oropharyngeal cancer: An observational study of diagnosis, prevalence and prognosis in a UK population. BMC Cancer 13: 220, 2013

2. Haeggblom L, Attoff T, Yu J, Holzhauser S, Vlastos A, Mirzae L, Ährlund-Richter A, Munck-Wikland E, Marklund L, Hammar stedt-Nordenvall L, et al: Changes in incidence and prevalence of human papillomavirus in tonsillar and base of tongue cancer during 2000-2016 in the Stockholm region and Sweden. Head Neck 41: 1583-1590, 2019.

3. Näsman A, Nordfors C, Holzhauser S, Vlastos A, Tertipis N, Hammar U, Hammarstedt-Nordenvall L, Marklund L, MunckWikland E, Ramqvist T, et al: Incidence of human papillomavirus positive tonsillar and base of tongue carcinoma: A stabilisation of an epidemic of viral induced carcinoma? Eur J Cancer 51: 55-61, 2015.

4. Chaturvedi AK, Engels EA, Pfeiffer RM, Hernandez BY, Xiao W, Kim E, Jiang B, Goodman MT, Sibug-Saber M, Cozen W, et al: Human papillomavirus and rising oropharyngeal cancer incidence in the United States. J Clin Oncol 29: 4294-4301, 2011.
5. Näsman A, Du J and Dalianis T: A global epidemic increase of an HPV-induced tonsil and tongue base cancer-potential benefit from a pan-gender use of HPV vaccine. J Intern Med 287: $134-152,2020$

6. Mellin H, Friesland S, Lewensohn R, Dalianis $\mathrm{T}$ and Munck-Wikland E: Human papillomavirus (HPV) DNA in tonsillar cancer: Clinical correlates, risk of relapse, and survival. Int J Cancer 89: 300-304, 2000.

7. Schache AG, Powell NG, Cuschieri KS, Robinson M, Leary S, Mehanna H, Rapozo D, Long A, Cubie H, Junor E, et al: HPV-related oropharynx cancer in the United Kingdom: An evolution in the understanding of disease etiology. Cancer Res 76: 6598-6606, 2016.

8. Schache AG, Simcock R, Gilbert DC and Shaw RJ: Changing face of HPV related cancer in the UK. BMJ 343: d6675, 2011.

9. Bersani C, Mints M, Tertipis N, Haeggblom L, Sivars L, Ährlund-Richter A, Vlastos A, Smedberg C, Grün N, Munck-Wikland E, et al: A model using concomitant markers for predicting outcome in human papillomavirus positive oropharyngeal cancer. Oral Oncol 68: 53-59, 2017.

10. Owadally W, Hurt C, Timmins H, Parsons E, Townsend S, Patterson J, Hutches on K, Powell N, Beasley M, Palaniappan N, et al: PATHOS: A phase II/III trial of risk-stratified, reduced intensity adjuvant treatment in patients undergoing transoral surgery for human papillomavirus (HPV) positive oropharyngeal cancer. BMC Cancer 15: 602, 2015.

11. Arenz A, Ziemann F, Mayer C, Wittig A, Dreffke K, Preising S, Wagner S, Klussmann JP, Engenhart-Cabillic R and Wittekindt C: Increased radiosensitivity of HPV-positive head and neck cancer cell lines due to cell cycle dysregulation and induction of apoptosis. Strahlenther Onkol 190: 839-846, 2014.

12. Kimple RJ, Smith MA, Blitzer GC, Torres AD, Martin JA, Yang RZ, Peet CR, Lorenz LD, Nickel KP, Klingelhutz AJ, et al: Enhanced radiation sensitivity in HPV-positive head and neck cancer. Cancer Res 73: 4791-4800, 2013.

13. Rieckmann T, Tribius S, Grob TJ, Meyer F, Busch CJ, Petersen C, Dikomey E and Kriegs M: HNSCC cell lines positive for HPV and p16 possess higher cellular radiosensitivity due to an impaired DSB repair capacity. Radiother Oncol 107: 242-246, 2013.

14. Vlashi E, Chen AM, Boyrie S, Yu G, Nguyen A, Brower PA, Hess CB and Pajonk F: Radiation-induced dedifferentiation of head and neck cancer cells into cancer stem cells depends on human papillomavirus status. Int J Radiat Oncol Biol Phys 94: 1198-1206, 2016.

15. Wang L, Wang X, Li Y, Han S, Zhu J, Wang X, Molkentine DP, Blanchard P, Yang Y, Zhang R, et al: Human papillomavirus status and the relative biological effectiveness of proton radiotherapy in head and neck cancer cells. Head Neck 39: 708-715, 2017.

16. Reid P, Wilson P, Li Y, Marcu LG, Staudacher AH, Brown MP and Bezak E: Experimental investigation of radiobiology in head and neck cancer cell lines as a function of HPV status, by MTT assay. Sci Rep 8: 7744, 2018.

17. Reid P, Wilson P, Li Y, Marcu LG, Staudacher AH, Brown MP and Bezak E: In vitro investigation of head and neck cancer stem cell proportions and their changes following X-ray irradiation as a function of HPV status. PLoS One 12: e0186186, 2017.

18. Pirotte EF, Holzhauser S, Owens D, Quine S, Al-Hussaini A, Christian AD, Giles PJ, Man ST, Evans M and Powell NG: Sensitivity to inhibition of DNA repair by olaparib in novel oropharyngeal cancer cell lines infected with human papillomavirus. PLoS One 13: e0207934, 2018.

19. Nordfors C, Vlastos A, Du J, Ahrlund-Richter A, Tertipis N, Grün N, Romanitan M, Haeggblom L, Roosaar A, Dahllöf G, et al: Human papillomavirus prevalence is high in oral samples of patients with tonsillar and base of tongue cancer. Oral Oncol 50: 491-497, 2014.

20. West CM, Davidson SE, Roberts SA and Hunter RD: Intrinsic radiosensitivity and prediction of patient response to radiotherapy for carcinoma of the cervix. Br J Cancer 68: 819-823, 1993.

21. Watson JV, Chambers SH and Smith PJ: A pragmatic approach to the analysis of DNA histograms with a definable G1 peak. Cytometry 8: 1-8, 1987.

22. Liao Y, Smyth GK and Shi W: FeatureCounts: An efficient general purpose program for assigning sequence reads to genomic features. Bioinformatics 30: 923-930, 2014.

23. Robinson JT, Thorvaldsdottir H, Winckler W, Guttman M, Lander ES, Getz G and Mesirov JP: Integrative genomics viewer. Nat Biotechnol 29: 24-26, 2011. 
24. Love MI, Huber W and Anders S: Moderated estimation of fold change and dispersion for RNA-seq data with DESeq2. Genome Biol 15: 550, 2014.

25. Benjamini Y and Hochberg Y: Controlling the false fiscovery rate: A practical and powerful approach to multiple testing. J $\mathrm{R}$ Stat Soc B 57: 289-300, 1995.

26. Krzywinski M, Schein J, Birol I, Connors J, Gascoyne R, Horsman D, Jones SJ and Marra MA: Circos: An information aesthetic for comparative genomics. Genome Res 19: 1639-1645, 2009.

27. Nulton TJ, Kim NK, DiNardo LJ, Morgan IM and Windle B: Patients with integrated HPV16 in head and neck cancer show poor survival. Oral Oncol 80: 52-55, 2018.

28. McBride AA and Warburton A: The role of integration in oncogenic progression of HPV-associated cancers. PLoS Pathog 13: e1006211, 2017.
29. Haeggblom L, Nordfors C, Tertipis N, Bersani C, Ramqvist T, Näsman A and Dalianis T: Effects of irradiation on human leukocyte antigen class I expression in human papillomavirus positive and negative base of tongue and mobile tongue squamous cell carcinoma cell lines. Int J Oncol 50: 1423-1430, 2017.

30. Spanos WC, Nowicki P, Lee DW, Hoover A, Hostager B, Gupta A, Anderson $\mathrm{ME}$ and Lee JH: Immune response during therapy with cisplatin or radiation for human papillomavirus-related head and neck cancer. Arch Otolaryngol Head Neck Surg 135: 1137-1146, 2009.

31. Vermeer DW, Spanos WC, Vermeer PD, Bruns AM, Lee KM and Lee JH: Radiation-induced loss of cell surface CD47 enhances immune-mediated clearance of human papillomavirus-positive cancer. Int J Cancer 133: 120-129, 2013. 